\title{
LA NOCIÓN DE PAISAJE EN ARQUEOLOGÍA. FORMAS DE ESTUDIO Y APORTES AL PATRIMONIO
}

\section{THE IDEA OF LANDSCAPE IN ARCHAEOLOGY. WAYS OF STUDING IT AND CONTRIBUTIONS TO HERITAGE}

\author{
Inés Gordillo ${ }^{1}$
}

\section{RESUMEN}

Este trabajo propone abordar la problemática del paisaje dentro del campo de la Arqueología, considerando las recientes perspectivas teórico-metodológicas y los aportes de otras disciplinas, así como el impacto recíproco entre estos avances y la gestión patrimonial. Para ello, es preciso transitar por la denominada Arqueología del Paisaje, en referencia al conjunto heterogéneo de estudios sobre la dimensión espacial caracterizados por una profunda reflexión crítica sobre el tema, junto con nuevas conceptualizaciones y vías de análisis del paisaje, centradas en la práctica social, percepción, la experiencia, el movimiento y la temporalidad. En este marco, son contemplados también algunos de los aportes decisivos de otras disciplinas, como la geografía humanista, la teoría de la estructuración social, la semiótica, la proxémica, la sintaxis espacial, etc. Para comprender más cabalmente la aplicación de los nuevos enfoques y métodos, son importantes en este trabajo los ejemplos y estudios de casos que muestran la manera de abordar integralmente aspectos centrales de la espacialidad, como la visibilidad, la circulación o la formación de paisajes culturales. Finalmente, se considera la influencia que estos enfoques han ejercido en el ámbito de la gestión patrimonial, así como el claro impacto de esta sobre el rol y las metas de la práctica arqueológica.

Palabras clave: Conceptos de Paisaje; Arqueología del Paisaje; Práctica social; Experiencia; Percepción; Análisis de visibilidad; Paisajes culturales; Gestión del patrimonio

\section{ABSTRACT}

The purpose of this paper is to approach the question of landscape within the field of archaeology taking into account recent theorical-methodological perspectives and the contributions of other disciplines, as well as the mutual impact between these advances and heritage management. In order to achieve this, we have to go through what is known as Landscape Archaeology, that is, the heterogeneous set of studies on spatial dimension defined by a deep critical reflection of the subject, together with new concepts and analysis paths to address landscape, focusing on social practice, perception, experience, movement and temporality. Within this context, other vital contributions of different disciplines, such as humanistic geography, social structuring theory, semiology, proxemics, spatial sintaxis, etc. are also

Tipología: Articulo de reflexión

Fecha de recepción: 27/02/2014

Fecha de aceptación: 31/07/2014

Como citar éste artículo: Gordillo, I. (2014). La noción de paisaje en arqueología. Formas de estudio y aportes al Patrimonio. Jangwa Pana, 13, 195 - 208

1. Instituto de Arqueología, Facultad de Filosofía y Letras, Universidad de Buenos Aires, Argentina. Correo: ibesalu@gmail.com. 


\section{ABSTRACT}

included in the analysis. The examples and case studies in this paper are important to fully understand the use of these new approaches and methods, since they show the way in which central aspects of spaciality, such as visibility, circulation or cultural landscapes formation, must be comprehensively addressed. Finally, this work identifies the influence of these approaches in heritage management as well as its clear impact over the rol and goals of archaeological practice.

Keywords: Concepts of Landscape; Landscape archaeology; Social practice; Experience; Perception; Analysis of visibility; Cultural Landscapes; Heritage management

\section{INTRODUCCIÓN}

$\mathrm{E}$ n estas líneas desarrollo la problemática expuesta en la conferencia magistral con la que se inauguró el IX Seminario Internacional de Conservación del Patrimonio "los retos de la revitalización en contextos multiculturales: paisajes, comunidades y espacios públicos" realizado en la Facultad de Humanidades de la Universidad del Magdalena a fines de noviembre de 2013. No fue un hecho casual que tal evento se haya abierto con la problemática del paisaje; por el contrario, responde claramente al interés e inquietud que el tema ha suscitado dentro de la gestión patrimonial, tanto en su esfera conceptual como en la metodológica y operativa. Una cuestión que se vio reflejada a lo largo de todo el Seminario a través de sus distintos actores y en referencia a los diversos escenarios de estudio y de acción.

En ese marco, mi compromiso fue presentar el concepto de paisaje dentro del campo de la arqueología actual, sus líneas de reflexión y herramientas analíticas -muchas de ellas derivadas de otras disciplinas sociales-, buscando ejemplos claros de su aplicación, para finalmente abrir la discusión sobre el impacto recíproco entre estos avances y la gestión patrimonial; cuestiones todas ellas que tránsito a lo largo de este texto.

Dentro de los estudios arqueológicos, durante las últimas décadas ha ganado terreno la denominada Arqueología del Paisaje, expresión que hace referencia a un conjunto de estudios sobre la dimensión espacial de las sociedades del pasado caracterizados por una potente crítica teórica sobre el tema junto con nuevas vías de análisis para su abordaje.

En términos generales, engloba a un conjunto heterogéneo de autores que otorgan particular énfasis al concepto de paisaje, dotándolo de nuevos significados. Muchos de estos autores, aunque no todos, pueden encuadrarse dentro de las corrientes post-procesuales de la arqueología. Pero, más allá de su posicionamiento teórico, estos recientes acercamientos a la dimensión espacial comparten intereses temáticos, ideas y métodos que se distancian de los estudios anteriores. Los une, además, un decidido cuestionamiento a la modernidad y la implementación de algunas propuestas teóricas y metodológicas procedentes de otras disciplinas sociales.

La crítica apunta hacia determinadas concepciones distintivas y explicativas del mundo que se desarrollaron durante la Edad Moderna. Con la revolución científica y el Iluminismo cristalizó en occidente una división categórica entre la Naturaleza y la Cultura, situando al paisaje dentro de la primera (Thomas 2001). El espacio moderno era concebido como "una realidad ya dada" y un contenedor de la acción del hombre que es medible en términos cartesianos y objetivos. A su vez, ese espacio "neutral" se encuentra divorciado de toda estructura de poder y dominación (Vaquer y Gordillo 2013). 
Estas ideas dominaron la historia científica occidental en distintos campos disciplinarios, y gran parte de la arqueología del siglo XX se desarrolló bajo su influencia. Si bien muchos enfoques de entonces le otorgaron a la esfera espacial una prioridad lógica en la interpretación de las sociedades del pasado, el paisaje fue conceptualizado como una entidad pasiva, en la cual los sistemas sociales del pasado dejan su impronta que a posteriori es interpretada por los arqueólogos con la expectativa de devolverle la "dinámica" que tuvo en su contexto sistémico. Desde esa óptica, el rol de los paisajes en la conducta humana es el de "telón de fondo", el marco dentro del cual las sociedades humanas desarrollan sus actividades de subsistencia (Vaquer y Gordillo 2013).

Los enfoques ecológicos y sistémicos de la llamada Nueva Arqueología, introdujeron un nutrido bagaje de métodos y técnicas para analizar el uso del espacio en términos adaptativos, con criterios que priorizaban las variables de ubicación y distancia a los recursos. Los modelos teóricos que subyacían a estos análisis espaciales, de carácter funcionalistas, adaptacionistas y/o economicistas, entendían que el ser humano toma siempre decisiones que minimizan los costes y maximizan los beneficios, reproduciendo, de esta manera, las lógicas de mercado que imperan en occidente (Soler Segura 2007).

Alejándose de tales explicaciones, desde los años noventa muchas de las investigaciones arqueológicas centradas en la espacialidad incorporan aspectos ignorados o desestimados previamente, como la percepción, la vivencia, el movimiento o la temporalidad. Se trata de conceptos largamente trabajados dentro de las Ciencias Sociales pero que no habían impactado decisivamente en la arqueología como sí ocurre con los nuevos enfoques en la materia. Veremos luego los principales aportes y la influencia que han ejercido estos otros campos disciplinarios en la Arqueología del Paisaje.
En ese contexto, también se destaca la aplicación de técnicas y métodos novedosos al estudio de los paisajes históricos, centrados en la visibilidad y otras formas de construcción social del paisaje, así como la aplicación de los Sistemas de Información Geográfica y otras herramientas preexistentes, reformuladas o re-orientadas dentro del nuevo marco conceptual sobre el paisaje.

Cabe señalar, finalmente, que tales reflexiones teóricas y herramientas metodológicas han ejercido una influencia estimulante en el ámbito de la gestión patrimonial, así como esta última ha impactado claramente sobre el rol y las metas de la práctica arqueológica.

\section{El concepto de paisaje en Arqueología}

Con los recientes enfoques sobre la problemática del paisaje y siendo este un criterio identificador que ahora aparece dotado de nuevos significados, la pregunta es entonces ¿qué es el paisaje?

Las nuevas propuestas apuntan a concebirlo en un sentido mucho más holístico y relacional. El primer paso en este terreno fue superar la concepción del espacio como algo dado, estático, de orden físico, reemplazándolo por una realidad social e históricamente construida, reconociendo su papel crítico en la dinámica social.

Christopher Tilley, uno de los impulsores de estos enfoques ${ }^{2}$, considera cuatro características básicas del paisaje: 1) es un concepto holístico, un conjunto de relaciones entre los lugares, 2) está constituido como conjuntos de relaciones espacio-temporales definidas por la vivencia, 3) es un medio primario de socialización y 4) es un recurso para la creación y reproducción del poder. La paradoja del paisaje, su dualidad, es que si bien es producido culturalmente, puede ser experimentado como algo ajeno a la producción

2. Este autor, junto con otros (Shanks, Thomas, Hamilton, Richards etc.) se orienta también hacia una arqueología de la percepción (fenomenológica), la que considera que se puede acceder al sentido original del registro arqueológico desde nuestra percepción actual de ese sentido. Se basa en la continuidad entre el ser y el percibir, el sujeto que percibe tiene de hecho una intuición del sentido del mundo (Tilley 1994 y 2008). 
humana. Y así, las redes de poder pueden ser legitimadas y parecer naturales (Tilley, 1996).

Cabe agregar además que, en estos términos, el espacio depende de quién lo experimenta y de cómo lo hace, ya que la experiencia espacial no es ni inocente ni neutra, sino que está condicionada por determinadas relaciones de poder que se estructuran en función de la edad, el género, la posición social, las relaciones económicas, etc. (Soler Segura, 2007). Es en este sentido que los paisajes son considerados como polisémicos (Bender, 1993; Thomas, 2001):

“...diferentes concepciones del paisaje pueden convivir al mismo tiempo. Ya sea como morada de los ancestros, como distribución de recursos o como lugar en el que se localizan sus hogares, el paisaje permite renovar la herencia ancestral, recolectar el alimento necesario para la subsistencia, crear lazos de identidad común, etc. Así, una misma realidad física adquiere, para un mismo colectivo humano, diversas formas y sentidos, los cuales están en constante construcción y reconstrucción." (Soler Segura, 2007: 52).

En otra línea paralela, Criado Boado (1993 y 1999) considera que las actividades en el espacio están organizadas de manera coherente con la representación del mundo que tiene el grupo social que las realiza. En el proceso de construcción de los espacios intervienen no sólo los dispositivos mecánicos, de orden físicos, sino también los dispositivos conceptuales, que definen, articulan y nombran. Desde la arqueología, analizando los primeros se debería poder llegar a los segundos $\mathrm{y}$, en definitiva, a su patrón de racionalidad. Sobre esa base, define al paisaje como producto sociocultural creado por la objetivación sobre el medio y en términos espaciales, de la acción social, tanto de carácter material como imaginario (Criado Boado, 1999).
Ahonda en la cuestión de la visibilidad ${ }^{3}$, buscando por ese medio un acercamiento a la racionalidad de cada grupo social. Las cuatro estrategias de visualización que este autor propone (inhibición, ocultación, exhibición y monumentalizarían) pueden relacionarse con una determinada racionalidad cultural, o incluso pueden emplearse como escala valorativa dentro del espacio construido. Propone además procedimientos muy sistematizados de estudio de los paisajes, distinguiendo e integrando distintas escalas de aproximación y análisis de diverso orden (formal, fisiográfico, de tránsito, de percepción, etc.). La corriente impulsada por Criado Boado en España y Latinoamérica, ha importantes producciones en diversos campos temáticos, que van desde los estudios de espacios arquitectónicos, agrarios o rupestres a distinta escala hasta la gestión del patrimonio.

Sintetizando lo expuesto, el enfoque conceptual propuesto por las arqueologías del paisaje sostiene la imposibilidad de separar la práctica social del paisaje, reconociendo en este último su carácter polisémico y su rol activo en la producción, reproducción y transformación sociales. El paisaje social, en su especificidad histórica, consiste en conjunto de relaciones entre sujetos y lugares que proporciona el contexto para la vida diaria, es una entidad relacional constituida por las personas en su compromiso con el mundo (Thomas, 2001). El paisaje se hace en tanto se vive, se piensa y se imagina, constituyéndose paralelamente como sistema de referencia en el que cada acción humana que se lleva a cabo es inteligible (Gosden \& Head, 1994)

Pero además de práctica y experiencia, el paisaje puede ser objeto y representación. Tiene una expresión formal y de allí la caracterización como "producto" sociocultural que Criado Boado le atribuye. $\mathrm{Y}$ esa materialización espacial de la

\footnotetext{
3. En realidad, se trata de dos tipos de análisis, complementarios entre sí, de lo que el autor denomina condiciones de visualización: 1) la visibilidad, lo que se ve desde un elemento arqueológico dado, 2) la visibilización, cómo se ve ese elemento concreto desde fuera de él y sobre el entorno (Criado Boado, 1999: 33)
} 
acción social de un grupo humano en el pasado -que puede ser transformada por una variedad de procesos y paisajes posteriores- cobra expresión actual en el registro arqueológico, el cual se transforma también en paisaje a partir de nuestra percepción, de nuestra mirada. Los paisajes arqueológicos o culturales del presente reúnen las expresiones pasadas, los productos de uno o más grupos humanos que allí habitaron, con las continuidades, rupturas, transformaciones y resignificaciones que se sucedieron en el tiempo, por lo que no puede reducirse a la suma de paisajes sucesivos que contiene.

En todos los casos, se trata de una representación históricamente constituida y factible de ser analizada en su proceso de conformación. Pero, para ello es fundamental reconocer que en la construcción de la espacialidad interviene de manera activa y sustancial la interpretación de los paisajes previos (Barrett, 1999; Ingold, 2000). Como resulta tan claro desde el campo del patrimonio, los paisajes -actuales y pretéritos- siempre contienen los restos materiales del pasado dentro de una red de interpretaciones coetáneas y sucesivas, por lo que su análisis arqueológico también permite abordar la manera en la que las sociedades se relacionaron y relacionan con su propio pasado (Vaquer \& Gordillo, 2013).

\section{Aportes de otras disciplinas}

Estas reflexiones sobre el paisaje, el lugar, la espacialidad, etc. no son exclusivas de los estudios arqueológicos. Más bien son producto del desarrollo de distintas disciplinas. Me detendré solo en algunos aportes que han impactado fuertemente en la Arqueología del paisaje.

En principio es preciso destacar la influencia ejercida por los geógrafos humanistas desde los años 70 y 80 . Esta influencia -no siempre reconocida en los estudios arqueológicos- ha sido fundamental para conceptualizar al paisaje en su carácter, antes mencionado, activo y holístico. Entre muchos, resalta la repercusión que ha tenido Yi Fu Tuan en, por ejemplo, arqueólogos como Christopher Tilley y Julian Thomas.

Tuan (1977), parte de una crítica a la noción científica occidental del espacio -propia de las corrientes positivistas y marxistas dominantes en la geografía- priorizando decididamente el rol de la experiencia desde una perspectiva conceptual ligada al existencialismo y la fenomenología. El lugar, el espacio y la experiencia son los conceptos claves de su argumentación. En principio, propone distinguir los dos primero, los cuales no son sinónimos pero se articulan de tal forma que no es posible comprender el uno sin el otro. El espacio es una entidad geométrica abstracta que se configura en lugar cuando los conocemos mejor y los dotamos de valor. Así, el lugar es una clase especial de objeto cargado de significados y el espacio es una red de lugares y objetos que las personas pueden experimentar directamente a través del movimiento y el desplazamiento, del sentido de dirección, de la localización relativa de objetos y lugares, y de la distancia que los separa y los relaciona (Delgado, 2003). En palabras de Tuan: "si consideramos al espacio como aquello que permite el movimiento, entonces el lugar es pausa; cada pausa en el movimiento hace posible que las locaciones se transformen en lugares" (Tuan, 1977: 6).

En la geografía humanística propuesta por Tuan, el cuerpo es el referente principal de la experiencia espacial y de la organización espacial del mundo (Delgado, 2003). Y la experiencia es considerada a través los modos directos -los sentidos- e indirectos -la simbolización- en que una persona conoce y construye la realidad. Y esa percepción siempre implica un componente emocional y subjetivo.

Esta perspectiva fue decisiva en la gestación de la arqueología fenomenológica, la que constituyó un avance hacia la deconstrucción de los paisajes de la modernidad burguesa, pero no estuvo exenta de críticas (Criado Boado, 1999; Vaquer, 2011) dado que traslada al pasado las percepciones de 
los agentes actuales, y de esta manera, reproducen la lógica que pretenden criticar y superar (Vaquer y Gordillo, 2013).

Por su parte, Edward Soja, desde el marxismo heterodoxo plantea reubicar la geografía dentro del contexto de la teoría social contemporánea enfocando el carácter espacial de la vida social reivindicado por las tendencias posmodernas. Su propuesta (Soja, 1985 y 2003) se centra en la espacialidad, un concepto que define como el espacio socialmente producido por el conjunto de las relaciones sociales, económicas, políticas y culturales entre los individuos y los grupos. La espacialidad existe en formas concretas de organización social y como medio propio de la vida social. La temporalidad de esta última, desde las rutinas y los eventos de la actividad cotidiana hasta la construcción de la historia a largo plazo, se articulan en la contingencia espacial e histórica.

Así, la espacialidad, la temporalidad y el ser social pueden ser consideradas las dimensiones que contienen todas las facetas de la existencia humana. Estas dimensiones existenciales cobran vida como construcciones sociales que moldean la realidad empírica al mismo tiempo que son moldeados por ella. Esta relación es concordante con la propuesta del antropólogo Tim Ingold (1993 y 2000) centrada en idea del habitar. Para este autor, la experiencia, modelada a partir de las interacciones diarias con los lugares es el resultado de realizar actividades, las que a su vez se desarrollan con diversas temporalidades. Este contexto relacional de involucrarse con el mundo a través de prácticas es habitar, y es lo que permite que los paisajes y los agentes se conformen mutuamente (Vaquer, 2011).

Desde esta perspectiva, no existe la distinción entre espacio -como algo abstracto- y lugar como algo significativo. Ingold (2000) considera que todos los espacios o lugares se encuentran cargados de significados, aún aquellos desconocidos, por lo que el "espacio" -en los términos de Tuanno existe; existen solamente nodos de lugares conectados entre sí por experiencias y narrativas (Vaquer y Gordillo, 2013). De igual modo, Thomas (2001) rechaza la idea de que un espacio abstracto, semánticamente indefinido, pueda ser transformado en un espacio significativo. Un lugar siempre se nos revela como un lugar; previamente no podemos tener conciencia de cualquier forma de no-lugar. El paisaje es, entonces, una red de sitios relacionados que han sido revelados e incorporados a la memoria mediante las interacciones y actividades habituales de las personas. Estas biografías personales y grupales están construidas de actos localizados, de allí la importancia de la red de sitios o lugares -el paisaje- en la constitución de la identidad (Thomas, 2001).

Al considerar el énfasis puesto en la relación entre las prácticas sociales y el paisaje, no podemos desconocer el impacto de la obra de sociólogos como Pierre Bourdieu y Anthony Giddens. La Teoría de la práctica o de estructuración social enfoca el carácter repetitivo de la vida social y la dependencia mutua de la estructura y el agente en términos de espaciotemporales. Las relaciones sociales se estructuran a través de prácticas sociales que se reiteran en el espacio y en el tiempo (Bourdieu, 1977; Giddens 1984).

Desde esta perspectiva, la rutina o repetición cotidiana de prácticas sociales posibilita la reflexividad del agente, pues si las prácticas sociales fuesen efímeras (y/o únicas) no sería posible el conocimiento por el sujeto del ambiente de actuación, imposibilitando su acción innovadora, creativa. Así, las características estructuradoras de los sistemas sociales tanto imponen restricciones a la acción como posibilitan que ésta se realice; esta es la dualidad de la estructura, caracterizada por la coerción y facilitación de la acción (Giddens, 1984). El espacio es así un instrumento de la acción social, juega un rol activo en la construcción social de la realidad, consideraciones que, como señalé antes, son emblemas 
de la arqueología del paisaje. Volvemos entonces a pensar el paisaje como un horizonte de inteligibilidad (Thomas, 2001) que brinda recursos para las prácticas y a su vez se encuentra estructurado por ellas.

Paralelamente, entre otros pensadores que han influenciado en los estudios arqueológicos sobre espacialidad se destaca Michel Foucault (1977), particularmente en referencia al paisaje arquitectónico. La línea de pensamiento que une la ideología del poder con la arquitectura tuvo un fuerte impulso con los aportes de este autor, especialmente a través del fascinante ejemplo del Panóptico, un edificio diseñado para inducir el estado de visibilidad consciente y permanente, asegurando el funcionamiento automático del poder. Para el caso, se trata de una íntima y explícita asociación entre ideología, mando social, y el ambiente construido. Para Foucault este es parte de las estrategias imperceptibles de la cultura material para formar individuos disciplinados y útiles. Resulta claro nuevamente que el paisaje, en este caso el paisaje arquitectónico, constituye un elemento crítico para la reproducción y coerción social.

Los paisajes arquitectónicos han recibido especial atención en Arqueología, tanto por su presencia en el registro como por su relevancia social. Si bien todas las dimensiones son intervenidas idealmente por el hombre, su acción material sólo afecta físicamente algunos espacios. La arquitectura genera lugares diferenciados mediante dispositivos técnicos y conceptuales, espacios cuyos caracteres materiales y simbólicos perfilan el rumbo de las actividades y la conducta social. A su vez, involucra al hombre en un espacio tridimensional acotado, vital en la formación de la existencia individual y colectiva. Por la importancia de su análisis, los arqueólogos hemos aplicado los aportes de una variedad de estudios y disciplinas, entre los que se destacan los referidos a la comunicación no verbal -semiótica y proxémica- y los análisis de sintaxis espacial.
Umberto Eco, por ejemplo, considera que la arquitectura presenta características de la comunicación de masas: es persuasiva y transmite mensajes subliminales, asimilados de manera inconsciente. De alguna manera se acerca la paradoja a Tilley (1996), antes mencionada, cuando plantea que el paisaje puede ser experimentado como algo ajeno a la producción humana y, de esa forma, legitima las redes de poder. Paralelamente, Eco considera que el discurso arquitectónico en su doble carácter denotativo y connotativo o simbólico, puede ser entendido solo cuando existen significados compartidos. La forma denota la función, la comunica y promueve, pero sólo con base en un sistema de hábitos y expectativas establecidas, es decir, con base en un código (Eco, 1984).

En muchos sentidos, la arquitectura puede ser entendida como un escenario montado para la comunicación. En esto cabe preguntarse también acerca del tamaño y organización del espacio construido y su relación con las posibilidades perceptivas humanas. Entramos así en el terreno de lo que se ha denominado Proxémica, frecuentemente empleada por los arqueólogos en diferentes contextos (Moore 1996; Sanders 1990; Hamilton et al., 2006; Gordillo 2005; entre otros).

Un pionero en este tipo de estudios fue el antropólogo Edward Hall. Su interés se centró en el análisis de los efectos de la distancia en la interacción interpersonal, postulando una serie de umbrales y límites impuestos por la fisiología de los sentidos humanos. Hall (1966 y 1972) define las distancias correspondientes a tales umbrales de comunicación, estructuradas por las habilidades de la visión, la voz y el oído, estableciendo parámetros y características para la comunicación íntima, personal, social y pública. Las críticas a la propuesta de Hall reconocen sus contribuciones, pero también sus fallas al observar que la conducta proxémica puede ser influida por algo más que los factores de distanciamiento (Altman, 1975; Sanders, 1990), como por ejemplo la con- 
dición social del individuo, los objetos semifijos; tabúes, prohibiciones, amenazas, la acción de los otros, los limites conceptuales, etc.

Desde otro ángulo, los análisis sobre Sintaxis Espacial ideados por los arquitectos Bill Hillier y Julienne Hanson permiten ordenar las relaciones entre las unidades espaciales para descubrir el patrón configuracional al que responden. Los espacios construidos son desglosados en componentes y analizados como redes, mediante la elaboración de mapas, gráficos e índices que muestran su grado de conectividad, integración y complejidad. Los métodos empleados, especialmente la elaboración de gráficos de accesos -también llamados modelos gamma- e índices derivados, solo requiere identificar espacios circunscriptos (recintos/nodos) y sus conexiones (accesos) (Hillier y Hanson 1984). Si bien se critica su carácter decididamente formal, en combinación con otras analíticas, ha sido aplicado con éxito en diversas investigaciones (Blanton, 1994; Moore, 1996; Mañana Borrazas, Blanco Rotea \& Ayan Vila 2002), contribuyendo a definir aspectos tales como permeabilidad, control de accesos, circulación, integración, complejidad, etc. del espacio arquitectónico.

\section{Formas de estudios del paisaje en Arqueología}

Me voy a detener particularmente en uno de estos trabajos que ilustra claramente muchas de estas aplicaciones. En el año 2002, Mañana Borrazas, Blanco Rotea y Ayan Vila formulan las bases teórico-metodológicas para una Arqueología de la Arquitectura, a la que denominan Arqueotectura. Se encuadran dentro de los lineamientos generales de la Arqueología del Paisaje desarrollada por el equipo de Criado Boado y pretenden superar los enfoques tradicionales en arquitectura incorporando herramientas conceptuales y operativas que permitan profundizar en el registro arquitectónico como un producto más de la cultura material y un mecanismo de reproducción del de sistemas sociales. Su propuesta presenta además una clara orientación patrimonial, resaltando el rol de la gestión, difusión de datos y puesta en valor.

Junto a los aportes teóricos propios de la arqueología postprocesual, estos autores integran los procedentes de otros campos del conocimiento como los mencionados previamente (teoría de la práctica, tecnologías de coerción, comunicación no verbal, sintaxis espacial, etc.). Consideran la multidimensionalidad del paisaje propuesta por Criado Boado (1999), buscando articular en su abordaje las dimensiones ambiental, social y simbólica. Para ello combinan varios tipos de estudios de los aspectos formales y perceptivos. El análisis formal incluye la deconstrucción y descripción de las unidades arquitectónicas en función de su forma geométrica y organización. Se trata de establecer relaciones formales del espacio construido, implementando mecanismo de zoom a distintas escalas, con el objetivo de obtener el modelo o patrón formal que subyace al paisaje arquitectónico. En el análisis formal comprende también al estratigráfico, orientado este último a identificar y ordenar los distintos momentos y los cambios formales a partir de la aplicación de técnicas arqueológicas como la matriz de Harris.

Paralelamente, se aplican los diagramas de permeabilidad o gráficos gamma (Hiller \& Hanson, 1984), cuantificando la comunicación, definiendo las posibilidades y tipo de acceso, los grados de privacidad de los espacios y la configuración del recorrido. Para el análisis de percepción se consideran las formas de circulación, su dirección y sentido, la identificación del hilo perceptivo, la proxémica, etc. En este marco, distinguen, como Criado Boado, el concepto de visibilidad y visibilización. El primero -la percepción des$d e$ - está referido a la situación del individuo que percibe, dentro y entre estructuras construidas, en un ángulo visual de $240^{\circ}$. La visibilización percepción de - alude a la visión de los volúmenes espaciales de manera estática, con la idea es identificar el orden y organización perceptiva de un espacio construido y sus relaciones internas y externas (si está imbuido en una panorámica 
abierta o cerrada, el juego de volúmenes, el impacto, la decoración, etc.).

En definitiva, se trata de un claro ejemplo de integración de los aportes interdisciplinarios que he considerado anteriormente que aplican exitosamente en el análisis de los castros y monumentos megalíticos de Galicia (Mañana Borrazas et al., 2002).

En este tipo de estudios la percepción, y particularmente la visibilidad, ocupan un lugar prioritario como vía de análisis de los paisajes sociales, en lógica coherencia con las premisas teóricas apuntadas en líneas anteriores. La visibilidad se constituye en una vía metodológica relevante debido a su potencial analítico para evidenciar relaciones poco visibles -valga la paradoja- en el registro arqueológico. Su estudio contribuye a identificar la lógica espacial que operó, individual o colectivamente, en un contexto arqueológico determinado.

Me he referido antes al énfasis metodológico que otorgan a la visibilidad los arqueólogos del paisaje, como el caso antes mencionado (Mañana Borrazás et al., 2002). Me interesa ahora considerar otro ejemplo, que se aleja de los estudios sobre megalitismo, arquitectura monumental o arte rupestre, $\tan$ frecuentes en este terreno. Sin embargo, este caso también permite valorar el análisis de visibilidad para reflexionar sobre la manera en que se articula la construcción de paisajes y relaciones sociales -como los lazos de vecindad y la vida aldeana-, aspectos que aparecerían ocultos en los estudios arqueológicos tradicionales. El trabajo en cuestión fue desarrollado por el equipo encabezado por Marcos Quesada en las sierras de El Alto-Ancasti ${ }^{4}$, un área ubicada en el borde oriental de la región andina del Noroeste

4. Se trata de un caso que conozco de cerca dado que en esa misma zona estamos desarrollando proyectos de investigación que abordan los espacios arquitectónicos, agrarios, rupestres, etc. bajo los lineamientos generales de la Arqueología del Paisaje y la aplicación de herramientas de análisis propias de la misma (análisis de percepción, circulación, SIG, etc.). Tales investigaciones también pueden tomarse para ilustrar esta exposición, pero por razones de espacio no me extiendo en ella (para más información consultar Gordillo, Zuccarelli \& Eguia, 2013). argentino. Tomaré aquí un artículo relativamente reciente sobre esas investigaciones (Quesada, Gastaldi \& Granizo, 2012) con el fin de mostrar algunas de las formas y procedimientos empleados en el estudio de la espacialidad.

Desde una perspectiva centrada en la experiencia de habitar (sensu Ingold 1993), los autores exploran el paisaje cotidiano a escala doméstica y comunitaria en una localidad de la sierras, conocida como El Taco, durante el primer milenio de La Era Cristiana. Buscan identificar formas concretas de construcción de las relaciones de vecin$\operatorname{dad}^{5}$ a partir de los modos en que ciertas prácticas habituales -tareas agrícolas, recorrido de sendas o percepción del entorno desde las viviendasgeneraron condiciones de inmediatez social.

Para este análisis, es decisivo considerar la topografía de la zona. El terreno se caracteriza por una alternancia de relieves positivos y negativos, con muchas pequeñas quebradas y lomadas que muestran evidencias de ocupación humana. La distribución de los conjuntos habitacionales no es homogénea debido a su ubicación en las explanadas elevadas. Esta lógica de instalación supuso una relativa separación entre los sitios de vivienda; el análisis de estadística espacial indica una tendencia a la dispersión ${ }^{6}$.

Ante este panorama, los autores indagan sobre la manera en que esta comunidad generó relaciones de vecindad sin la conformación de núcleos aldeanos más compactos. Consideran que esas relaciones no implican necesariamente cortas distancias entre los sitios, sino la posibilidad de sus habitantes de establecer instancias cotidianas de interacción que podrían caracterizar la inmediatez de la vida aldeana.

5. Siguiendo a Appadurai (2001, citado por Quesada et al., 2012) los autores consideran las formas en que se constituyen las relaciones sociales a escala local, como son el sentido de la inmediatez social, las tecnologías de interacción social y la relatividad de los contextos de copresencia). 6. Análisis de Distancia Promedio al Vecino más Cercano (Average Nearest Neighbor Distance) mediante el software $\mathrm{ArcGis}^{\mathrm{TM}}$ arrojó un valor de $318,7 \mathrm{~m}$ (mayor a distancia media esperada de $333,8 \mathrm{~m}$ ), siendo el índice de vecino más cercano de 1,25 (Quesada et al. 2012). 
Desde esta perspectiva, la noción de aldea deja de estar directamente vinculada a la cantidad y proximidad de viviendas para enfocar otras formas de vecindario que generan una alta frecuencia de interacciones sociales entre vecinos. La proximidad no sería entonces más que uno de estos recursos que, al parecer, fue usado en el área manera bastante limitada. En cambio, parece que otros medios fueron los que posibilitaron las relaciones de vecindad entre viviendas más distantes (Quesada et al., 2012). Es aquí donde cobra importancia el análisis de visibilidad, y más específicamente el referido al fenómeno de intervisibilidad.

Veamos entonces de qué se trata. La ubicación elevada de los conjuntos habitacionales hace que resulten visibles entre sí. Este fenómeno de intervisibilidad fue objeto de un análisis detallado a través de la determinación de las cuencas visuales correspondientes a cada uno de los sitios de vivienda ${ }^{7}$, lo que permitió reconocer la intensidad de la relación (ver Quesada et al., 2012, figura 10: 16). Sobre esta base:

"Podemos imaginar entonces que ver a los vecinos, aún a la distancia, formaba parte de la experiencia cotidiana de los habitantes de estos conjuntos de habitación y también que esta experiencia debió ser más intensa mientras las casas estaban habitadas ya que los muros, que sabemos que eran bastante elevados, las techumbres y el humo de los hogares pudieron haber hecho de estas viviendas elementos más destacados del paisaje." (Quesada et al., 2012: 16)

Paralelamente, otro aspecto significativo es que hay una marcada relación entre las líneas de circulación y la localización de los conjuntos de habitación. Las sendas elevadas recorren las dorsales topográficas conectando las explanadas elevadas y al hacerlo también van comunican-

7. El análisis de Cuencas Visuales (Viewshed), que define las superficies del terreno visibles desde una o más localizaciones en función de la topografía, fue realizado mediante el software ArcGis ${ }^{\mathrm{TM}}$ (Quesada et al., 2012). do los conjuntos de habitación dispuestos sobre ellas (ver Quesada et al., 2012, figura 10: 16). Los análisis cuali-cuantitativos de circulación muestran claramente que las casas se disponen en cercanías de las sendas y, de esa forma, es posible que el desplazamiento por ese territorio haya sido también una ocasión de interacción con las familias que habitaban las demás casas conectadas por las sendas.

De esta forma se caracteriza al paisaje arqueológico de la zona dando cuenta de los elementos que lo integran y la manera en que éstos se vinculan, pero también, y principalmente, indagando acerca de los recursos materiales mediante los cuales se conformaban las relaciones sociales de vecindad. Se trata de un paisaje campesino cuyos principales componentes eran las viviendas, los espacios agrícolas aterrazados y las sendas que permitían atravesar la quebrada geografía local. Allí, las tecnologías de interacción basadas en la intervisibilidad y la circulación posibilitaban instancias de encuentro, de inmediatez social o de contextos de co-presencia generando una forma particular de vecindario donde verse y visitarse parecen haber sido situaciones propiciadas por la misma estructuración del paisaje (Quesada et al., 2012).

En un sentido más general, este tipo de abordaje nos abre la posibilidad de formas distintas de pensar la espacialidad. El paisaje social que acabamos de mostrar se distancia de aquella visión neutral o estática de la dimensión espacial, tan cuestionada por las perspectivas actuales del paisaje. También nos desafía a considerar otros recursos o componentes en la construcción de los paisajes locales, despojándonos de la comodidad de imaginar sólo concentraciones, límites o monumentos en nuestra mirada al pasado.

\section{Paisaje y gestión patrimonial}

Finalmente, las formulaciones conceptuales y las herramientas operativas procedentes de los estudios recientes del paisaje han influenciado 
sobre el escenario de la gestión patrimonial. En las últimas décadas se fue ampliando de manera progresiva el concepto de Patrimonio, el cual actualmente incorpora desde sitios históricos particulares hasta el paisaje como unidad, concebido desde una visión holística y relacional.

Al respecto, la Convención del Patrimonio Mundial en 1992 promovió la determinación de paisajes culturales como una categoría aparte merecedora de reconocimiento y protección. En ese marco, el término "paisaje cultural" incluye una diversidad de manifestaciones de la interacción entre el hombre y su ambiente natural, que comprenden distintas categorías de paisajes culturales ${ }^{8}$.

Por su parte, la Convención Europea del Paisaje en 2000 rechaza la idea del paisaje como simple realidad física y valoriza en su concepción al componente subjetivo, considerando que es la percepción/interpretación humana la que define el paisaje (Ballesteros et al., 2005). Esa misma Convención también abre la posibilidad de calificar como Paisajes Culturales no sólo enclaves de características "excepcionales" (bióticas o abióticas) sino también espacios cotidianos o degradados.

Asimismo, como afirma Abejez, la visión integradora que subyace a la noción de Paisajes Cultuales, contempla también a la ciudad como un conjunto que reúne las construcciones, los

8. Las categorías de paisajes culturales definidas por la Convención del Patrimonio Mundial en 1992 son: 1) Los paisajes claramente definidos, diseñados y creados intencionalmente por el hombre, como los jardines y los parques; 2) Los paisajes evolutivos (u orgánicamente desarrollados) resultantes de condicionantes sociales, económicas, administrativas y/o religiosas, que se han desarrollado conjuntamente y en relación con su medio ambiente natural. Se dividen en dos subcategorías: a- paisaje fósil / relicto, es aquél en el que un proceso de evolución concluyó en algún momento del pasado, pero sus rasgos significativos son todavía visibles materialmente y b- paisaje activo, continuo en el tiempo, que sigue teniendo un papel social activo en la sociedad contemporánea, conjuntamente con la forma tradicional de vida; $\mathrm{Al}$ mismo tiempo, posee huellas materiales significativas de su evolución a lo largo del tiempo; 3) Los paisajes asociativos de los aspectos religiosos, artísticos o culturales relacionados con los elementos del medio ambiente. Son aquellos en los que existen poderosas asociaciones religiosas, artísticas o culturales del elemento natural en lugar de pruebas culturales materiales, que pueden ser insignificantes o incluso no existir. espacios, los restos arqueológicos e, incluso, el contexto natural y social, la que ha permitido superar - siguiendo las recomendaciones del Memorandum de Viena de 2005 - los conceptos clásicos de centros históricos y de conjuntos monumentales, pudiéndose hablar con propiedad de la existencia de un "paisaje urbano", aunando un conjunto heterogéneo aunque indisoluble y continuo a lo largo del tiempo, pues incluso las discontinuidades forman parte del proceso histórico, permitiendo superar la dicotomía naturaleza/cultura tanto como la de pasado/presente (Abejez, 2013: 5-6).

En forma recíproca, muchos arqueólogos vinculados a la gestión patrimonial tienden a integrar las reflexiones surgidas en las convenciones de instituciones tales como la UNESCO o la Unión Europea. En ese sentido, se ha generado un diálogo tan fluido que, actualmente, cualquier proyecto de investigación sobre el paisaje pretérito presenta conexiones directas con la labor patrimonial (Soler Segura, 2007). De hecho, muchos de los investigadores que reflexionan teóricamente sobre el paisaje en Arqueología participan en programas orientados a la gestión del patrimonio ${ }^{9}$.

Entre los casos notables de esta interacción entre ambos campos de acción, está dado por la línea de investigación del Laboratorio de Arqueología del Paisaje (LAr) de Santiago de Compostela, desde donde se entiende el paisaje arqueológico como una de esas manifestaciones de la acción social que, al ser observadas, dan lugar a un Paisaje Cultural. Desde hace más de dos décadas vienen proclamando la necesidad de incorporar la gestión de patrimonio en los proyectos y programas arqueológicos, sobre la base de un acercamiento al paisaje arqueológico como paisaje cultural. Entre los muchos trabajos que en ese terreno el LAr ha producido, los proyec-

9. En mi caso particular, que durante muchos años me he dedicado a la investigación básica, los proyectos en lo que actualmente participo contemplan acciones vinculadas a la gestión de patrimonio, comenzando por la construcción de espacios de interacción (talleres, recorridos conjuntos, participación en trabajos comunes, actividades en escuelas, etc.) que permitan compartir, discutir y evaluar con las comunidades locales los resultados de las investigaciones hasta proyectos de puesta en valor y turismo. 
tos para la conservación y puesta en valor de la Sierra de Suído, resultan muy ilustrativos. Según Ballesteros Arias, Otero Vilariño y Varela Pousa (2005). observamos allí un paisaje cultural que es consecuencia de la acción de las actividades humanas en diferentes épocas a los largo de 13.000 años. Se integran en ese paisaje abrigos rocosos del Paleolítico y Epipaleolítico, grupos de túmulos megalíticos del Neolítico, grabados vinculados Edad del Bronce, yacimientos de época castreña y evidencias de ocupación romana, plena ocupación de los valles en época medieval e inicios de la implantación de una economía ganadera que continúa en la Edad Moderna, y un momento actual que apunta a una transformación sustancial del paisaje anterior. Durante esta larga trayectoria no hubo una destrucción del paisaje preexistentes para la construcción de uno nuevo, si no que el nuevo usó y recreó lo que ya estaba hecho. Ejemplo de ese fenómeno - sin duda frecuente en muchos otros contextos- es el de los túmulos megalíticos que pasan a formar parte del propio paisaje de sierra; no desaparecen, sino que se incorporan al mundo simbólico de las sociedades siguientes, bien como hitos en el paisaje, como referentes de leyendas, de topónimos, etc. (Ballesteros et al., 2005). En síntesis, el área se nos presenta como una superposición e integración de diferentes paisajes sociales que abarca los primeros momentos de instalación humana y las subsiguientes fases de ocupación sin la ocultación y/o destrucción del paisaje diseñado anteriormente. Un tipo de paisaje cultural que, según la Convención Mundial de la UNESCO, estaría englobado dentro de la categoría de un Paisaje evolutivo y orgánicamente desarrollado, que se ha desarrollado hasta su forma actual (Ballesteros et al., 2005). También es un claro ejemplo de la conceptualización -antes considerada- del paisaje como una representación históricamente constituida que puede ser analizada en su proceso de conformación, reconociendo que la capacidad y efecto de interpretar los paisajes previos por parte de los distintos sujetos y grupos a través del tiempo es un factor relevante en la construcción de la espacialidad.

\section{A modo de cierre}

Muchos autores observan que la tendencia actual dentro de la gestión patrimonial es concebir al paisaje como la unidad mínima de intervención, desplazando el interés en la conservación de elementos o sitios aislados hacia la protección íntegra de los paisajes culturales (Ruiz Zapatero 1998; Soler Segura, 2007; Abejez 2013). En ese sentido, las recientes perspectivas teóricas de la arqueología y otras disciplinas sociales contribuyen a no concebir erróneamente a los paisajes culturales como un resultado directo de las acciones de los grupos humanos sobre su entorno natural ni como una simple estratigráfica cultural, apuntando en cambio hacia una visión integrada de lugares, prácticas e interpretaciones que se estructuraron en distintos planos y lógicas espaciales dentro de un mismo territorio. Y en ese marco, la arqueología ofrece vías descriptivas y analíticas decididamente rentables para abordar la gestión patrimonial de los paisajes, especialmente los análisis cuali-cuantitativos de visibilidad, tránsito y de los Sistemas de Información Geográficos.

Pero además, siguiendo a Ballesteros et al. (2005), la noción de Paisajes Culturales requiere trabajar en la protección y difusión del patrimonio a una escala más amplia, desde estrategias que contemplen la complejidad, problemática y posibilidades del paisaje. En este sentido, la tarea del arqueólogo no puede ser tan sólo una búsqueda de la memoria o la identidad (op cit: 7). Los avances en el campo del patrimonio ofrecen un nuevo papel para la arqueología, aportando criterios para la identificación, caracterización, valoración y gestión de los paisajes culturales. Una tarea que no puede realizarse sin la interacción y el acuerdo de los diferentes actores y disciplinas involucrados en el tema (pobladores locales, especialistas, políticos, planificadores, etc.) con sus diferentes intereses, objetivos y aportes. Desde allí, es necesario analizar la vulnerabilidad de los paisajes culturales y trazar estrategias y políticas de actuación en ellos, evaluando previamente sus 
consecuencias. En este escenario, los estudios de impacto se constituyen como otro de los principales aportes de la arqueología a la gestión patrimonial de los Paisajes Culturales.

\section{REFERENCIAS BIBLIOGRÁFICAS}

Abejez, L. (2013). Arqueología en los espacios urbanos. La gestión integral del Patrimonio de las ciudades (ms).

Altman, I. (1975). The environment and social behavior: Privacy, personal space, territoriality and crowding. Monterey, California, Brooks/ Cole Publish Company.

Ballesteros A. P., Otero Vilariño, C., \& Varela, P. R. (2005). Los Paisajes Culturales desde la arqueología: propuestas para su evaluación, caracterización y puesta en valor. ArqueoWeb 7(2) Recuperado de: http://pendientedemigracion.ucm.es/info/arqueoweb/pdf/7-2/ballesteros.pdf

Barrett, J. C. (1999). Defining Domestic Space in the Bronze Age of Southern Britain. En: M. Parker Pearson and C. Richards (Eds), Architecture and Order. Approaches to Social Space (pp. 87-97). London, Rutledge.

Blanton, Richard, 1994. Houses and Households: A Comparative Study, Plenum Press.

Bender, B. (1993) 'Introduction: Landscape, Meaning and Action. En: B. Bender (Ed.). Landscape: Politics and Perspectives, pp. 1-17. Oxford: Berg.

Bourdieu, P. (1977). Outline of a Theory of Practice. Cambridge University Press. Cambridge.

Criado Boado, F. (1993). Límites y Posibilidades de la Arqueología del Paisaje. Revista de Prehistoria y Arqueología, No 2, pp. 9-55.

Criado Boado, F. (1999). Del terreno al espacio: Planteamientos y perspectivas de la Arqueología del Paisaje. CAPA 6: 1-82.

Delgado, O. (2003) Debates sobre el espacio en la geografia contemporánea. Universidad Nacional de Colombia. Bogotá: Unibiblos.
Eco, U. (1984). Función y signo: La semiótica de la arquitectura. En: El lenguaje de la arquitectura. México: Editorial Limusa.

Foucault, M. (1977). Vigilar y Castigar. Buenos Aires: Siglo XXI Editores.

Giddens, A. (1984). La constitución de la sociedad: bases para la teoría de la estructuración. Buenos Aires: Amorrortu Editores.

Gordillo, I. (2005). Arquitectos del rito. La construcción del espacio público en La Rinconada, Catamarca. Relaciones de la Sociedad Argentina de Antropología, tomo XXIX, pp. 111-136.

Gordillo, I., Zuccarelli, V., \& Eguia, L. (2013). Las casas del sol naciente. Arqueología de la vertiente oriental de El Alto-Ancasti. En: B. Ventura, M. B. Cremonte \& M. G. Ortiz (Eds). Arqueología y Etnohistoria de la vertiente oriental de los Andes de Argentina y Bolivia. En prensa.

Gosden, O \& Herad L. (1994). Landscape - a usefully ambiguous concept", Archaeology in Oceania, 29, pp. 113-116. Hall, E. (1966). The Hidden Dimensions. Doubleday, Garden City, New York.

Hall, E (1972). Silent assumptions in social communication. En: R. Gutman (Ed.) People and Building (pp. 135-151). Basic Books, New York.

Hamilton, S; R. Whitehouse; K. Brown; P. Combes; E. Herring \& Seager Thomas, M. 2006). Phenomenology in Practice: Towards a p Methodology for a 'Subjective' Approach. European Journal of Archaeology, pp. 9-31.

Hillier, B. \& J. Hanson (1984). The Social Logic of Space. Cambridge University Press, Cambridge.

Ingold, T. (1993). The Temporality of the Landscape. World Archaeology, 25 (2), pp: 152-174.

Ingold, T. (2000). The Perception of the Environment. London, Routledge.

Mañana Borrazás, P.; Blanco Rotea, R. \& Ayán Vila, X. M. (2002). Arqueotectura 1: Bases teórico-metodológicas para una arqueología de la arquitectura. TAPA 25, pp. 1-103. 
Moore J. (1996). Architecture and power in the Ancient Andes. The Archaeology of public buildings. Cambridge University Press. Cambridge.

Quesada, M.; Gastaldi, M. \& Granizo M. G. (2012). Construcción de periferias y producción de lo local en las cumbres de EL AltoAncasti. Relaciones 37 (2): 435-456.

Sanders, D. (1990). Behavioral Conventions and Archaeology: Methods for the Analysis of Ancient Architecture. En: S. Kent (Ed.), Domestic Architecture and Use of Space. An interdisciplinary cross-cultural study. New Directions in Archaeology (pp. 21-59) Cambridge University Press. Cambridge.

Soja, E. (1985). The Spatiality of Social Life: Towards a Transformative Retheorisation. En D. Gregory and J. Urry (Eds) Social Relations and Spatial Structures (pp. 90-127). London, MacMillan Publishers.

Soja, E. (2003). Postmodern Geographies. The Reassertion of Space in Critical Social Theory (Octava Edición), Verso Londres y Nueva York. Soler Segura, J. (2007). Redefiniendo el registro material. Implicaciones recientes desde la Arqueología del Paisaje anglosajona. Trabajos de Prehistoria, 64 (1), pp.: 41-64.
Thomas, J. (2001). Archaeologies of Place and Landscape. En: I. Hodder(Ed.), Archaeological Theory Today (pp. 165-186). Polity Press, Cambridge.

Tilley, C. (1994): A phenomenology of landscape. Places, Paths and Monuments, Oxford. Tilley, C. (1996). The power of the rocks: topography and monument construction on Bodmin Moor. En: R. Bradley (Ed.), Sacred Geography. World Archaeology 28, No 2 (pp. 167-175), London.

Tilley, C. (2008). Phenomenological Approaches to Landscape Archaeology. En: B. David y J. Thomas (Eds). Handbook of Landscape Archaeology (pp. 271 - 276). Left Coast Press. California.

Tuan, Y.(1977). Space and Place. The Perspective of Experience. University of Minnesota Press. Minneapolis.

Vaquer, J. M. (2011) Paisaje y prácticas sociales en Cruz Vinto. Una interpretación desde los espacios domésticos externos. Editorial Académica Española, Saarbrücken.

Vaquer J. M. y I. Gordillo (2013). Recorriendo los Paisajes. En: I. Gordillo y J. Vaquer (Eds) La espacialidad en arqueología. Enfoques, métodos y aplicación. Ed. Abya Yala. Quito. 